\title{
Use of Six Sigma Methodology to Reduce Appointment Lead-Time in Obstetrics Outpatient Department
}

Ortíz Barrios, Miguel Angel, Felizzola Jiménez, Heriberto

\begin{abstract}
This paper focuses on the issue of longer appointment lead-time in the obstetrics outpatient department of a maternal-child hospital in Colombia. Because of extended appointment lead-time, women with high-risk pregnancy could develop severe complications in their health status and put their babies at risk. This problem was detected through a project selection process explained in this article and to solve it, Six Sigma methodology has been used. First, the process was defined through a SIPOC diagram to identify its input and output variables.

Second, six sigma performance indicators were calculated to establish the process baseline. Then, a fishbone diagram was used to determine the possible causes of the problem. These causes were validated with the aid of correlation analysis and other statistical tools. Later, improvement strategies were designed to reduce appointment lead-time in this department. Project results evidenced that average appointment lead-time reduced from 6,89 days to 4,08 days and the deviation standard dropped from 1,57 days to 1,24 days. In this way, the hospital will serve pregnant women faster, which represents a risk reduction of perinatal and maternal mortality.
\end{abstract}

\section{Keywords}

Healthcare, Outpatient, ObstetricsSix Sigma 